\title{
COETUM. PROYECTO PARA LA CREACIÓN DE UN ESPACIO DE ÁGORA EDUCATIVA. EVALUACIÓN DE UN PROYECTO DE INNOVACIÓN PARA LA TRANSFORMACIÓN DE LA FORMACIÓN INICIAL DEL PROFESORADO
}

\section{COETUM. THE CREATION OF AN EDUCATIONAL AGORA SPACE PROJECT. INNOVATION PROJECT ASSESSMENT FOR THE INITIAL TEACHER TRAINING TRANSFORMATION}

http://dx.doi.org/10.15304/ie.28.5188

\author{
Víctor Valdés Sánchez \\ vvaldess@alumnos.unex.es \\ Prudencia Gutiérrez Esteban \\ Universidad de Extremadura \\ pruden@unex.es
}

\section{RESUMEN}

El artículo presenta el proyecto COETUM, creado bajo el marco de la convocatoria de acciones de innovación docente del Servicio de Orientación y Formación Docente de la Universidad de Extremadura, cuyo principal objetivo es habilitar un espacio educativo que permita nuevas formas de organización y sirva de punto de encuentro para alumnado y profesorado. En la primera parte, se reflexiona acerca de la importancia que tienen los espacios en educación. En la segunda, se describe el proyecto, así como las acciones desarrolladas. En la tercera parte, se lleva a cabo un análisis del mismo mediante el uso de formulario en línea y la realización de un grupo de discusión y por último, se extraen algunas ideas finales sobre la experiencia desarrollada.

Palabras clave: Formación del profesorado, Entorno educativo, innovación educativa, enfoque multidisciplinario, metodología docente.

\section{ABSTRACT}

This article presents the COETUM project, created under the framework of the call for innovation actions of teaching innovation of the Service of Orientation and Teacher Training of the University of Extremadura. whose main objective is to enable an educational space that allows new forms of organization and serves as a point meeting for students and teachers. In the first part, we reflect on the importance of spaces in education. In the second, the project is described, as well as the actions developed. In the third part, an analysis of it is carried out through the use of online form and the realization of a discussion group and finally, some final ideas about the experience developed are extracted.

Keywords: Teacher Training, Educational Environment, Educational Innovation, Multidisciplinary Approach, Teaching Methodology. 


\section{INTRODUCCIÓN}

La vinculación y relación de las personas con el entorno es una constante a lo largo de la historia que ha influido en el estado físico, cognitivo y de manera general en el desarrollo de las personas (Remess, y Winfield, 2008; Acar, 2014; Amann, 2016). Es por esta influencia que también ha dejado su huella en muchísimas corrientes y metodologías educativas como apuntan Olivos (2010) o Muñoz-Rodríguez y Olmos (2016).

De esta forma, la gestión y el uso de los espacios ha sido una demanda recurrente del profesorado en Educación que no ha sido resuelta a lo largo de la historia, dado que en muchos aspectos como es la concepción de escuela inclusiva, la normativa que regula las edifícaciones escolares a la que hace referencia Crespo y Lorenzo (2016) no tiene interiorizada aún la interconexión entre ambiente y persona.

De este modo, se han perpetuado escenarios educativos que no favorecen un adecuado desarrollo de metodologías que difieran de la clase expositiva. Thornburg (2004), Ultralab (2004), Heppell, Chapman, Millwood, Constable y Furness, (2004), Cohen (2010) y Rute (2010) proponen un replanteamiento del espacio educativo desde cuatro cualidades con las que todo edificio educativo debe contar. Así, deben ser espacios flexibles, inspiradores, que supongan un apoyo para el aprendizaje y envolventes. Los nuevos modelos educativos vienen demandando la renovación de los lugares de aprendizaje y la utilización de las zonas comunes de los centros educativos para actividades de aprendizaje para dar respuesta a las diferentes metodologías, enfoques y teorías del aprendizaje como argumentan Rigolon (2010), Guldbaek, Vinkel y Broens (2011) o Guney y Al (2012).

Así, Boettcher (2007), Kuuskorpi y Cabellos-González (2011), Mokthar, Jiménez Rodríguez, Hepell y Segovia (2016) abogan por entender los espacios como un instrumento educativo. Para ello se debe tener en cuenta, siguiendo la propuesta de Eberhard (2009), la distribución espacial, los elementos que los ocupan, su funcionalidad, las proporciones, la iluminación, la ubicación, así como la relación que guardan con el exterior. Todos estos elementos pueden incidir en los resultados académicos, en los niveles de estrés y en las reacciones que se generan en el cerebro.

Dentro de estos planteamientos existe una gran diversidad de posibilidades de estructuración del espacio en función de la modalidad del aprendizaje que ha recogido Hernando Calvo (2015), existiendo así entre otros, espacios presenciales horizontales como rincones, laboratorios y ateliers ligados con metodologías ABS o Design Thinking que fomentan un aprendizaje cooperativo. En cuanto a los espacios digitales, nos dan la oportunidad de extender la experiencia educativa fuera del aula, pero también a traer el mundo exterior a clase, de fusionar los ambientes formales e informales, gracias a las smart schools (Ibrahim, Razak y Kenayathulla, 2013) y el aprendizaje invisible (Cobo y Moravec, 2010).

En el caso de este proyecto, se alude a la concepción del espacio como un ágora educativa. Se trata, como argumentan Fernández-Díaz, Gutiérrez-Esteban y Fernández Olaskoaga (2015), de diseñar espacios que posibiliten la transformación de la dinámica habitual de transmisión unidireccional del conocimiento en el aula universitaria, diversificando el espacio de trabajo y enriqueciendo el intercambio de conocimiento entre el alumnado. 


\section{COETUM. PROYECTO PARA LA CREACIÓN DE UN ESPACIO DE ÁGORA EDUCATIVA}

El proyecto COETUM nace financiado dentro de la convocatoria pública competitiva de proyectos de innovación del Servicio de Orientación y Formación Docente (SOFD) de la Universidad de Extremadura durante el curso 2016-2017, con el objetivo de habilitar un espacio educativo que permita nuevas formas de organización del aula y sirva de punto de encuentro para el alumnado y profesorado de la facultad. De esta forma los objetivos que se plantean para el proyecto son los siguientes:

1. Habilitar un espacio educativo que permita nuevas formas de organización del aula y sirva de punto de encuentro para el alumnado y el profesorado de la facultad.

2. Favorecer la presencia de las escuelas en el contexto universitario y acompañar la innovación en el ámbito escolar creando puentes entre la Universidad y la Escuela.

3. Desarrollar la participación del alumnado universitario en el diseño de procesos de innovación educativa y visibilizar otras posibilidades metodológicas del aula universitaria.

4. Fomentar una actitud participativa, crítica y activa entre el alumnado de la Facultad de Educación.

5. Colaborar con otras redes universitarias para intercambiar experiencias en un marco más amplio de relación interuniversitaria.

6. Divulgar en formato de publicaciones, congresos o jornadas los resultados del proyecto.

En cuanto a las personas destinatarias del proyecto, son el alumnado y profesorado de la Facultad de Educación. Actualmente la Facultad se encuentra conformada por cerca de 2190 estudiantes (lo que la sitúa como uno de los centros de mayor tamaño de la UEx) y alrededor de 120 docentes y 23 miembros del personal de administración y servicios que hacen posible la enorme actividad diaria que vive el centro en el que se imparten, como se ha podido constatar, tres Títulos de Grado (cuatro grupos de Ed. Primaria, dos de Ed. Infantil y uno de Psicología) y otros cinco de Máster, más el alumnado proveniente de la Universidad de Mayores y de los programas de doctorado que se ofertan actualmente en la facultad.

Sobre el lugar de desarrollo del proyecto, aunque en un primer momento se planteó que estuviese al aire libre; finalmente, atendiendo a las condiciones meteorológicas, se decidió ubicarlo en el patio interior de la facultad. Se trata de un espacio polivalente, dotado de mesas, sillas y bancos; un punto de encuentro utilizado sobre todo por el alumnado, para la realización de trabajos en grupo, como lugar de descanso e incluso como zona de estudio. Este espacio también cuenta con máquinas expendedoras de refrescos y café, así como un microondas y un frigorífico. Se escogió este lugar atendiendo primordialmente a las condiciones climáticas, a la vez que se trataba de un lugar visible dentro de la facultad y de un espacio donde el alumnado se siente cómodo, lo cual es garantía de éxito del proyecto.

Debido a la amplitud del proyecto, tanto en número de personas implicadas, como en la variedad de campos de conocimiento y titulaciones, se establecen siete líneas de trabajo para mantener una mayor organización en las actividades llevadas a cabo. Dichas líneas son creación, decoración e instalación del ágora, ágora como punto de encuentro, clases dentro del ágora, acciones formativas conjuntas intracentro, intercambio entre alumnado de grado de otras universidades, 
intercambio y experiencias con escuelas y formación del profesorado participante en el proyecto. Cada una de éstas se describen a continuación.

\section{Creación, decoración e instalación del ágora}

Supone la creación de una estructura semicircular que permita el diálogo y la comunicación entre las personas participantes con capacidad para acoger un grupo de estudiantes de grado de la Facultad de Educación. Una vez creada la estructura, tiene lugar el proceso de decoración del ágora, siendo una oportunidad para trabajar contenidos de asignaturas específicas así como para mantener el espacio vivo y cuidado.

\section{2. Ágora como punto de encuentro}

Se trata de hacer un uso transversal del ágora, convirtiéndolo en un lugar de encuentro para el alumnado, profesorado y personal de la facultad. Un espacio cómodo y atractivo que sirva para generar relaciones y conversaciones en torno a la educación y que pueda dinamizar la vida del centro.

\section{Clases dentro del ágora}

El desarrollo de las clases dentro del ágora supone la actividad central del proyecto. Da la posibilidad al profesorado de llevar a cabo sus clases en un espacio diferentes con múltiples posibilidades; sobre todo, ligadas a la comunicación y la interacción entre el alumnado. Un ejemplo de este tipo de actividades serían los debates, las actividades en grupo y las exposiciones de trabajos.

\section{Acciones formativas conjuntas intracentro}

Se trata de llevar a cabo actividades conjuntas vinculadas a asignaturas, materias, competencias de grado o máster, de manera que planifiquen y desarrollen acciones formativas conjuntas, dirigidas al alumnado universitario, desde varias asignaturas de un mismo grado para trabajar determinadas competencias transversales o generales.

\section{Intercambio entre alumnado de grado de otras universidades}

En consonancia con las experiencias de intercambio e interacción anteriores con alumnado de grado de las facultades de Educación de la Universidad de Cantabria y la Universidad del País Vasco-EHU, se apuesta por continuar esta línea de trabajo donde se aboga por la difusión en medios digitales de las producciones del alumnado (redes sociales, YouTube, Google Drive), en el contexto de las asignaturas implicadas en el proyecto, basadas en el diseño de actividades conjuntas o bien complementarias, que puedan ser trabajadas desde distintas materias/semestres/cursos de las tres universidades. El alumnado de una universidad las difunde y recibe una evaluación acerca de las tareas realizadas por estudiantes de otra universidad mediante el desarrollo de ágoras digitales intercentros.

\section{Intercambio y experiencia con escuelas}

Intercambio y desarrollo de actividades conjuntas con centros educativos de la zona. Diseño de actividades y materiales educativos (por parte del alumnado de grado) para el desarrollo de dichas actividades para el alumnado de Ed. Primaria y Ed. Infantil. Jornada-Convivencia de intercambio en la Facultad de Educación. Pero también, que sea un lugar de encuentro con profesorado en activo de las mismas. Propiciar el intercambio mediante el desarrollo de seminarios, actividades y acciones colaborativas con centros educativos. 


\section{Formación al profesorado participante en el proyecto}

Todo proceso de innovación requiere de la formación del profesorado para poder desarrollar de la mejor manera el proyecto. Por este motivo, se cuenta con una línea de formación transversal en la que se cuenta con especialistas en metodologías innovadoras en Educación para que den soporte a las actividades mediante la creación de talleres formativos.

Tras describir las líneas principales del proyecto, se exponen en la tabla 1 las actividades llevadas a cabo, el impacto de cada una de ellas en referencia al número de destinatarios que recibieron la actividad y su correspondencia con la tipología y metodología utilizadas.

\begin{tabular}{|c|c|c|c|}
\hline Título de la actividad y descripción & Tipología & Metodología & $\mathrm{N}^{0}$ part. \\
\hline $\begin{array}{l}\text { El espacio de las Humanidades en los horizontes sociales y educativos: Se abordaron } \\
\text { dos cuestiones diferenciadas. Por un lado, el arrinconamiento de los conocimientos } \\
\text { sociales. Por otro lado, el proceso ideológico y didáctico en el sistema educativo. }\end{array}$ & $\begin{array}{l}\text { Acciones formativas } \\
\text { conjuntas intracentro }\end{array}$ & $\begin{array}{l}\text { Clase } \\
\text { magistral }\end{array}$ & 60 \\
\hline $\begin{array}{l}\text { El profesorado del siglo XXI: debate a partir de artículos relacionados con el tema de } \\
\text { la sesión. Se decoró el Ágora con frases extraídas de dichos artículos. }\end{array}$ & $\begin{array}{l}\text { Ágora como punto de } \\
\text { encuentro }\end{array}$ & Debate & 15 \\
\hline $\begin{array}{l}\text { Separación de gemelares en el aula: Estudio de caso desde la perspectiva del } \\
\text { peritaje psicológico judicial: Presentación del caso, Estructura del informe pericial, } \\
\text { Metodología, Valoración, Resultados y Consideraciones finales }\end{array}$ & $\begin{array}{l}\text { Clases e intercambio en } \\
\text { el ágora }\end{array}$ & $\begin{array}{l}\text { Clase } \\
\text { magistral }\end{array}$ & 64 \\
\hline $\begin{array}{l}\text { Elaboración de Posters de autores de psicología de la educación: Trabajo en equipo } \\
\text { para la creación de poster de divulgación. }\end{array}$ & $\begin{array}{l}\text { Ágora como punto de } \\
\text { encuentro }\end{array}$ & \begin{tabular}{l|} 
Trabajo en \\
pequeño grupo
\end{tabular} & 137 \\
\hline $\begin{array}{l}\text { Almorzando con imágenes y vídeos: Reflexión a partir de los relatos narrativos del } \\
\text { alumnado de la UPV-EHU y de la UEx en formato vídeo y/o imagen. }\end{array}$ & $\begin{array}{l}\text { Intercambio entre alumnado } \\
\text { de otras universidades }\end{array}$ & Debate & 100 \\
\hline $\begin{array}{l}\text { Debate sobre el modelo actual de Orientación Educativa en Extremadura: dos grupos } \\
\text { de debate entre aspectos a favor y en contra de la orientación especializada interna } \\
\text { (en el centro) o externa (equipo de sector). }\end{array}$ & $\begin{array}{l}\text { Clases e intercambio en el } \\
\text { ágora }\end{array}$ & Debate & 30 \\
\hline $\begin{array}{l}\text { Diseño de decoración del Ágora: Descripción del proyecto, documentación, diseño, } \\
\text { presupuesto y realización. }\end{array}$ & $\begin{array}{l}\text { Creación, instalación y } \\
\text { decoración del ágora }\end{array}$ & $\mathrm{ABP}$ & 19 \\
\hline $\begin{array}{l}\text { Acercando caminos: Invitación a profesorado en activo experto en temática } \\
\text { relacionada con la materia. }\end{array}$ & $\begin{array}{l}\text { Intercambio y experiencias } \\
\text { con escuelas }\end{array}$ & Debate & 40 \\
\hline $\begin{array}{l}\text { Conocimiento y reinterpretación de una obra de arte: Análisis y estudio de una obra } \\
\text { pictórica de las vanguardias históricas y reinterpretación con materiales escolares. }\end{array}$ & $\begin{array}{l}\text { Creación, instalación y } \\
\text { decoración del ágora }\end{array}$ & Taller & 170 \\
\hline $\begin{array}{l}\text { Conocemos la radio escolar del CEIP Ciudad de Badajoz: Análisis del trabajo que } \\
\text { desde el centro se hace en la radio escolar y de los diferentes programas de radio } \\
\text { grabados este curso académico. }\end{array}$ & $\begin{array}{l}\text { Intercambio y experiencias } \\
\text { con escuelas }\end{array}$ & \begin{tabular}{l|} 
Trabajo en \\
pequeño grupo
\end{tabular} & 50 \\
\hline $\begin{array}{l}\text { Resolución de situaciones problemáticas a través de la programación y la robótica: } \\
\text { Programación y Robótica Educativa Orígenes. La actualidad de la robótica en } \\
\text { Educación Infantil y Primaria. }\end{array}$ & $\begin{array}{l}\text { Ágora como punto de } \\
\text { encuentro }\end{array}$ & $\begin{array}{l}\text { Resolución de } \\
\text { problemas }\end{array}$ & 9 \\
\hline $\begin{array}{l}\text { Igualdad para la prevención de la violencia de género a través de manifestaciones } \\
\text { plásticas: Conferencia con un espacio de debate-taller. }\end{array}$ & $\begin{array}{l}\text { Acciones formativas } \\
\text { conjuntas intracentro }\end{array}$ & Conferencia & 33 \\
\hline $\begin{array}{l}\text { Decoración del Ágora a través de instalaciones artísticas: Creación de hormigas de } \\
\text { cartón para colocarlas en las paredes del ágora. }\end{array}$ & $\begin{array}{l}\text { Creación, instalación y } \\
\text { decoración del ágora }\end{array}$ & Taller & 130 \\
\hline $\begin{array}{l}\text { Calculando en la época romana: actividad para aprender a calcular con números } \\
\text { romanos utilizando el ábaco. }\end{array}$ & $\begin{array}{l}\text { Clases e intercambio en el } \\
\text { ágora }\end{array}$ & \begin{tabular}{|l|} 
Clase \\
magistral \\
\end{tabular} & 50 \\
\hline Musicoterapia: Sesión introductoria del uso de la musicoterapia en educación & $\begin{array}{l}\text { Ágora como punto de } \\
\text { encuentro }\end{array}$ & Taller & 25 \\
\hline $\begin{array}{l}\text { Narrativa y creatividad en la universidad ¿es posible transitar otros caminos en } \\
\text { la enseñanza y el aprendizaje?": Sesión formativa teórico-práctica de un profesor } \\
\text { especialista. }\end{array}$ & $\begin{array}{l}\text { Formación del profesorado } \\
\text { participante en el proyecto }\end{array}$ & \begin{tabular}{|l} 
Relatos \\
narrativos
\end{tabular} & 10 \\
\hline
\end{tabular}

Tabla 1. Actividades del proyecto y número de participantes. 
Como se aprecia en la tabla anterior, el proyecto acogió un total de dieciséis actividades diferentes que tuvieron lugar a lo largo de todo el curso académico 2016-2017. El alcance del proyecto en cuanto a la participación de los destinatarios ha sido de entorno a 900 personas vinculadas a estudios de Grado en Educación Infantil, Primaria y Psicología. Estudios de máster de investigación, de formación del profesorado de Educación secundaria y de orientación educativa. La participación mínima ha sido de nueve personas y la participación máxima de ciento setenta personas.

Haciendo una lectura diferente de las actividades en relación con las líneas de trabajo anteriormente descritas, el gráfico 1 muestra el peso de cada una de las líneas de trabajo del proyecto. El ágora como punto de encuentro ha sido la línea que ha contado con más actividades, seguida de las clases dentro del ágora y la creación, instalación y decoración del ágora. Por el contrario, la formación del profesorado y el intercambio con otras escuelas fueron las líneas menos desarrolladas. Aún así, se percibe una distribución más o menos igualitaria entre todas las líneas del proyecto.

Tipología de Actividades

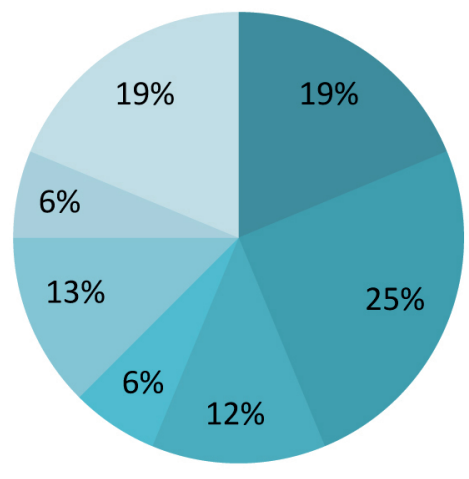

- Creación, instalación y decoración del ágora

- Ágora como punto de encuentro

Acciones formativas conjuntas intracentro

- Intercambio entre alumnado de grado de otras universidades

- Intercambio y experiencias con escuelas

Formación al profesorado participante en el proyecto

Clases e intercambio en el ágora

Gráfico 1. Tipología de las actividades del proyecto.

Respecto a la opción metodológica, el proyecto contemplaba no solo el cambio del espacio donde tiene lugar la formación, sino también realizar cambios metodológicos que permitan mejorar los resultados de aprendizaje. En este sentido, en el gráfico 2, se aprecian las diferentes metodologías puestas en práctica por el profesorado participante en el proyecto. En total se han aplicado nueve metodologías diferentes con la peculiaridad que en algunas de las actividades se han aplicado dos tipos de metodología diferente. Tratándose de un proyecto de ágora es lógico que la metodología más usada sea el debate, seguida de la clase magistral que a pesar de no ser la metodología que se pretendía aplicar, en la mayoría de los casos se ha combinado con alguna otra metodología de carácter más participativo o activo. 


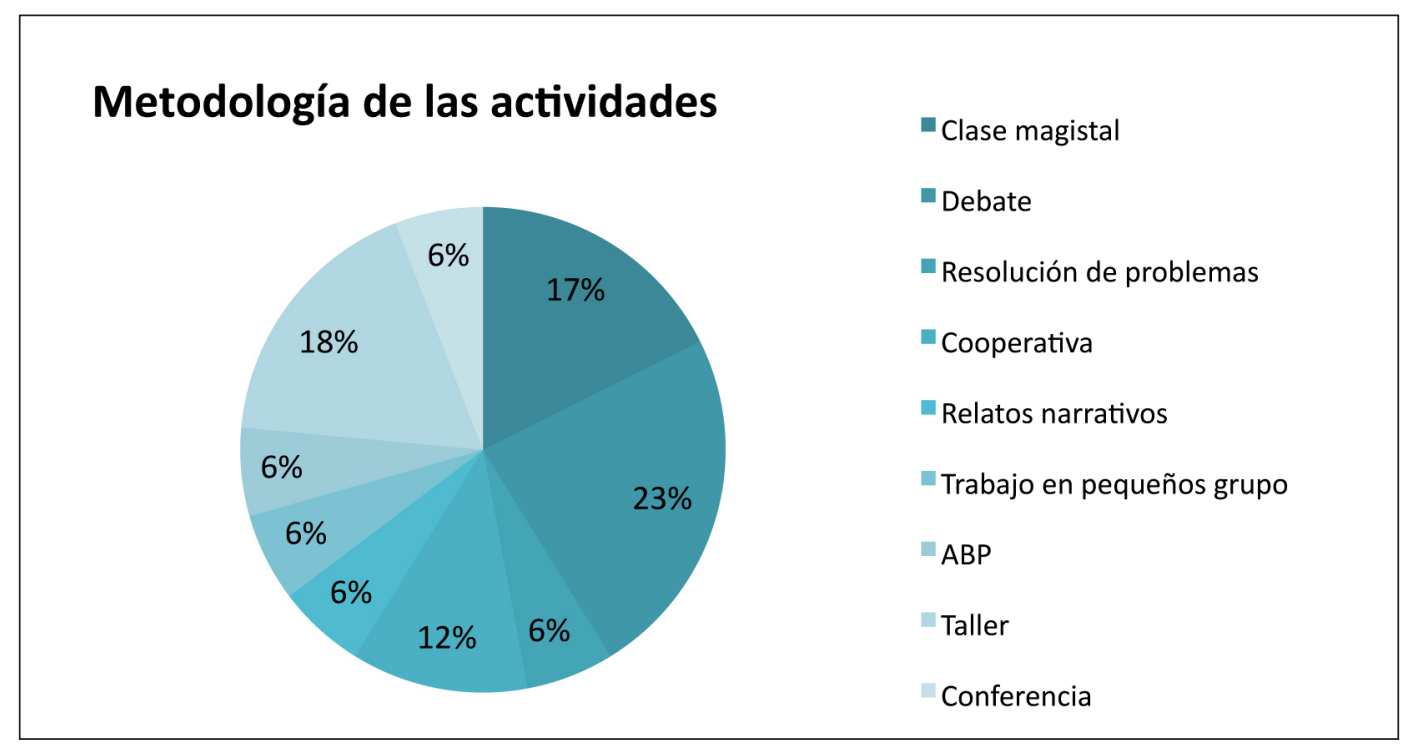

Gráfico 2. Metodologías empleadas en el proyecto.

En síntesis, el proyecto ha hecho posible innovar en cuanto a cuatro ejes que están vinculados con el proceso enseñanza-aprendizaje: el lugar donde se desarrolla la actividad, la metodología docente empleada, los recursos y el rol del alumnado y profesorado en la relación educativa.

\section{MÉTODO}

\section{Participantes}

En el proyecto participaron 23 personas, 21 profesoras y profesores universitarios de las Universidades de Extremadura, Cantabria y País Vasco y 2 alumnos de doctorado de la Universidad de Extremadura. En la tabla 2 se muestra la diversidad de perfiles participantes en el proyecto. Cabe destacar que se trata de un proyecto que cuenta con la participación de profesionales participantes de cuatro departamentos diferentes y abarca un total de ocho áreas de conocimiento, lo cual le otorga al proyecto un carácter transversal y convergente que no suele ser muy común. 


\begin{tabular}{|c|c|c|c|c|c|c|c|c|}
\hline $\begin{array}{l}\text { Total } \\
\text { Participantes }\end{array}$ & \multicolumn{2}{|c|}{ Sexo } & \multicolumn{2}{|l|}{ Departamento } & \multicolumn{2}{|l|}{ Área } & \multicolumn{2}{|l|}{ Universidad } \\
\hline \multirow{10}{*}{23} & \multirow{5}{*}{ M } & \multirow{5}{*}{14} & $\begin{array}{l}\text { Ciencias de la } \\
\text { Educación }\end{array}$ & 8 & $\begin{array}{l}\text { Didáctica y Organización } \\
\text { Escolar }\end{array}$ & 6 & \multirow[t]{2}{*}{$\begin{array}{l}\text { Universidad de } \\
\text { Extremadura }\end{array}$} & \multirow[t]{2}{*}{21} \\
\hline & & & Didáctica CC. & & & & & \\
\hline & & & $\begin{array}{l}\text { Experim. y } \\
\text { Matemáticas }\end{array}$ & 2 & Didáctica de las Matemáticas & 2 & \multirow{4}{*}{$\begin{array}{l}\text { Universidad de } \\
\text { Cantabria }\end{array}$} & \multirow{4}{*}{1} \\
\hline & & & $\begin{array}{l}\text { Psicología y } \\
\text { Antropología }\end{array}$ & 3 & $\begin{array}{l}\text { Psicología Evolutiva y de la } \\
\text { Educación }\end{array}$ & 2 & & \\
\hline & & & $\begin{array}{l}\text { Didáctica CC. } \\
\text { Sociales, Lengua } \\
\text { y Literatura }\end{array}$ & 5 & $\begin{array}{l}\text { Didáctica de la Lengua y la } \\
\text { Literatura }\end{array}$ & 2 & & \\
\hline & \multirow{5}{*}{$\mathrm{H}$} & \multirow{5}{*}{9} & \multirow{4}{*}{$\begin{array}{l}\text { Didáctica Exp. } \\
\text { Musical, Plástica } \\
\text { y Corporal }\end{array}$} & \multirow{3}{*}{3} & $\begin{array}{l}\text { Didáctica de las Ciencias } \\
\text { Sociales }\end{array}$ & 3 & & \\
\hline & & & & & $\begin{array}{l}\text { Didáctica de la Expresión } \\
\text { Plástica }\end{array}$ & 3 & \multirow{4}{*}{$\begin{array}{l}\text { Universidad del } \\
\text { País Vasco }\end{array}$} & \multirow{4}{*}{1} \\
\hline & & & & & $\begin{array}{l}\text { Métodos de Investigación y } \\
\text { Diagnóstico en Educación }\end{array}$ & 2 & & \\
\hline & & & & & $\begin{array}{l}\text { Personalidad, Evaluación y } \\
\text { Tratamiento Psicológico }\end{array}$ & 1 & & \\
\hline & & & $\begin{array}{l}\text { Programa de } \\
\text { doctorado }\end{array}$ & 2 & $\begin{array}{l}\text { Doctorado en Innovación en } \\
\text { Formación del Profesorado. } \\
\text { Asesoramiento Análisis de la } \\
\text { Práctica Educativa y TIC en } \\
\text { Educación }\end{array}$ & 2 & & \\
\hline
\end{tabular}

Tabla 2. Relación de perfiles participantes en el proyecto.

\section{Instrumentos}

Para evaluar la experiencia del proyecto de innovación se utilizó un cuestionario en línea, a través de un formulario en Google Form, donde el profesorado pudo volcar todos los datos de cada actividad, así como valoraciones personales acerca del proyecto. Para complementar al cuestionario, se diseñó, además, un grupo de discusión para evaluar con mayor profundidad la experiencia, utilizando una técnica más acorde a las metodologías vinculadas al proyecto. En la en la tabla 3 se observa la participación de las personas participantes en cada una de las dos iniciativas.

\begin{tabular}{|c|c|c|}
\hline MÉTODO DE EVALUACIÓN & N $^{\mathbf{0}}$ PARTICIPANTES & PORCENTAJE \\
\hline Formulario Google & $19 / 23$ & $83 \%$ \\
\hline Grupo de discusión & $5 / 23$ & $22 \%$ \\
\hline
\end{tabular}

Tabla 3. Métodos de evaluación y participación. 
La tabla 3 muestra una participación alta en el cuestionario, alcanzando el $83 \%$ de la participación total, pero en cambio se percibe una baja participación en el grupo de discusión que tan solo contó con el $22 \%$ de las personas participantes.

\section{Procedimiento}

Para la categorización de los datos recabados se utilizó un criterio temático, de manera que se identifican las referencias que hablan de un mismo tema a lo largo de las respuestas del formulario y el grupo de discusión. Asimismo, en la tabla 4, se agrupan las referencias, las cuales se elevan en categorías, siempre en función de la similitud temática. La distribución de las referencias en las diferentes categorías así como el posterior análisis, se ha llevado a cabo de manera manual.

\begin{tabular}{|l|c|c|}
\hline CATEGORÍAS & $\begin{array}{c}\text { No DE REFERENCIAS POR } \\
\text { CATEGORÍA }\end{array}$ & \multirow{2}{*}{ No TOTAL DE REFERENCIAS $^{\mathbf{0}}$} \\
\cline { 1 - 2 } VALORACIONES POSITIVAS & 12 & \multirow{2}{*}{4} \\
\cline { 1 - 2 } VALORACIONES NEGATIVAS & 2 \\
\cline { 1 - 2 } DIFICULTADES DEL PROYECTO & 4 \\
\cline { 1 - 2 } PROPUESTAS DE MEJORA & 8 & \\
\cline { 1 - 2 } CONTINUIDAD DEL PROYECTO & 6 & \\
\cline { 1 - 2 } RESULTADOS DEL PROYECTO & 16 & \\
\hline
\end{tabular}

Tabla 4. Categorías y referencias.

\section{RESULTADOS}

De manera inductiva se han identificado 48 referencias, elevadas a seis categorías diferentes que abarcan desde las valoraciones del proyecto hasta los resultados del proyecto o las propuestas de mejora del mismo. Cabe destacar que son las categorías de resultados y valoraciones positivas donde mayor número de referencias se han encontrado. A continuación, se desarrolla en profundidad el análisis interpretativo de cada una de las categorías que han emergido.

En relación a las valoraciones positivas del proyecto que aparecen reflejadas en la tabla 5, se destacan varios elementos fundamentales como son el carácter multidisciplinar que ha impregnado el proyecto: "haya participado tantísima gente y de tantas áreas de conocimiento"; el rol del alumnado en el desarrollo de esta experiencia, pues ha participado y se ha comprometido de manera voluntaria con el proyecto. Para el alumnado estas actividades han resultado muy satisfactorias, habida cuenta de que aumentó la motivación en muchas de las asignaturas implicadas en el proyecto; y por último la oportunidad de desarrollar una reflexión mucho más profunda del aprendizaje que ha venido de la mano de este proyecto: "una oportunidad de debate y reflexión que va más allá del temario establecido." 


\begin{tabular}{|l|l|l|l|}
\hline CATEGORÍA & $\begin{array}{c}\text { No DE } \\
\text { REFERENCIAS }\end{array}$ & PARTICIPANTE & REFERENCIAS \\
\hline \multirow{2}{*}{$\begin{array}{l}\text { VALORACIONES } \\
\text { POSITIVAS }\end{array}$} & Docent4. FORM & $\begin{array}{l}\text { "El proyecto nos ha parecido ciertamente muy interesante, } \\
\text { al igual que la formación recibida. Es muy motivador el } \\
\text { hecho de conocer de primera mano otras metodologías y } \\
\text { formas de trabajar en el aula universitaria." }\end{array}$ \\
\cline { 2 - 4 } & Docent6. G.D & $\begin{array}{l}\text { "Esta experiencia ha sido aleccionadora y significativa } \\
\text { y el hecho de que haya participado tantísima gente y de } \\
\text { tantas áreas de conocimiento pues ha sido para mí muy } \\
\text { enriquecedor, me ha gustado mucho." }\end{array}$ \\
\cline { 2 - 4 } & Docent8. FORM & $\begin{array}{l}\text { "La experiencia fue muy positiva, al unir a dos profesores } \\
\text { del Departamento con intereses comunes y transformar } \\
\text { por un día el espacio Ágora y la dinámica en las aulas en } \\
\text { una oportunidad de debate y reflexión que va más allá del } \\
\text { temario establecido." }\end{array}$ \\
\hline
\end{tabular}

Tabla 5. Referencias referidas a valoraciones positivas.

En la tabla 6 se presentan las valoraciones negativas. En relación a las dos únicas valoraciones negativas que ha recibido el proyecto por parte del profesorado participante, estas inciden en la falta de recursos materiales e infraestructuras que han limitado el desarrollo del proyecto por la falta de dotación económica del mismo. De la misma manera, se atribuye la falta de coordinación entre el profesorado participante a las limitaciones horarias y a la elevada carga de trabajo.

\begin{tabular}{|l|c|l|l|l|}
\hline CATEGORÍA & $\begin{array}{c}\mathbf{N}^{0} \text { DE } \\
\text { REFERENCIAS }\end{array}$ & PARTICIPANTE & REFERENCIAS \\
\hline \multirow{2}{*}{$\begin{array}{l}\text { VALORACIONES } \\
\text { NEGATIVAS }\end{array}$} & 2 & Docent5. FORM & $\begin{array}{l}\text { "Experiencia negativa en cuanto a recursos e } \\
\text { infraestructuras" }\end{array}$ \\
\cline { 2 - 4 } & Docent1. FORM & $\begin{array}{l}\text { "No ha funcionado todo lo deseado pues había dificultades } \\
\text { en la coordinación entre las profesoras participantes por } \\
\text { las limitaciones horarias y de carga de trabajo." }\end{array}$ \\
\hline
\end{tabular}

Tabla 6. Referencias referidas a valoraciones negativas.

En consonancia con la valoración negativa en cuanto a la falta de coordinación, en la tabla 7 se destaca la dificultad que ha encontrado el profesorado para atender el proyecto debido a que suponía un sobreesfuerzo el sumar al elevado volumen de trabajo y carga lectiva, el trabajo colaborativo con el resto de profesorado participante que suponía la participación en un proyecto de estas características, a pesar de su predisposición a ello.

Además, de nuevo en la tabla 7 se alude a la problemática que ha supuesto para el profesorado no acostumbrado al empleo de metodologías activas en el aula, vincular el contenido de sus asignaturas con el trabajo en el ágora sintiéndose cómodo con el resultado. Hay que destacar que ha supuesto para gran parte del profesorado participante la primera experiencia alejada de la clase magistral con todo lo que ello conlleva. 


\begin{tabular}{|c|c|c|c|}
\hline CATEGORÍA & $\begin{array}{c}\mathrm{N}^{0} \mathrm{DE} \\
\text { REFERENCIAS }\end{array}$ & PARTICIPANTE & REFERENCIAS \\
\hline \multirow{2}{*}{$\begin{array}{l}\text { DIFICULTADES } \\
\text { DEL PROYECTO }\end{array}$} & \multirow[t]{2}{*}{4} & Docent1. G.D & $\begin{array}{l}\text { "El problema que yo veo sinceramente es que no tenemos } \\
\text { tiempo en nuestro horario para hacer cosas porque no } \\
\text { coincidimos. (...) En este centro tenemos un volumen de } \\
\text { trabajo muy grande porque tenemos muchos estudiantes, } \\
\text { muchas asignaturas y yo de verdad no he visto falta de } \\
\text { voluntad en nadie, porque todo el mundo no puede venir y } \\
\text { escribe, se disculpa, que no es que digas es que pasan, es un } \\
\text { tema de disponibilidad." }\end{array}$ \\
\hline & & Docent4. G.D & $\begin{array}{l}\text { "La principal dificultad venía por conectar con lo que } \\
\text { yo estaba haciendo en el aula en el sentido de... A mí por } \\
\text { ejemplo el uso del ágora lo tengo más interiorizado con un } \\
\text { tipo de actividad de reflexión, de debate, entonces conectar } \\
\text { esto con lo que tú estabas haciendo normalmente en las clases } \\
\text { para que no fuera como un pegote, no fuera una cosa de me } \\
\text { tengo que ir abajo." }\end{array}$ \\
\hline
\end{tabular}

Tabla 7. Referencias referidas a dificultades del proyecto.

De cara a la mejora del proyecto y de nuevo en relación a las valoraciones negativas, en la tabla 8 se proponen la remodelación y mejora del espacio habilitado como ágora para hacerlo más atractivo, hecho que no ha sido posible realizarse debido a la falta de dotación económica del proyecto en esta convocatoria.

De nuevo con la intención de mejorar las condiciones de trabajo a las que se enfrenta el profesorado que innova y que está dispuesto a afrontar esta clase de proyectos, en la tabla 8 se solicita un espacio horario común al profesorado que sirva para atender el trabajo colaborativo que se desprende de esta clase de iniciativas.

\begin{tabular}{|c|c|c|c|}
\hline CATEGORÍA & $\begin{array}{c}\mathrm{N}^{0} \mathrm{DE} \\
\text { REFERENCIAS }\end{array}$ & PARTICIPANTE & REFERENCIAS \\
\hline \multirow{3}{*}{$\begin{array}{l}\text { PROPUESTAS } \\
\text { DE MEJORA }\end{array}$} & \multirow[b]{3}{*}{8} & Docent10. FORM & $\begin{array}{l}\text { "Nos habría gustado que se pudiera llevar a cabo la } \\
\text { remodelación del espacio, el presupuesto era mínimo. Solo se } \\
\text { pudo realizar el proyecto en vídeo." }\end{array}$ \\
\hline & & Docent4. G.D & $\begin{array}{l}\text { "Hacerlo como más que anime a las propias actividades, } \\
\text { el espacio también cuenta mucho. Un espacio que sea más } \\
\text { cómodo, más bonito." }\end{array}$ \\
\hline & & Docent1. G.D & $\begin{array}{l}\text { "Podemos pedir para el año que viene, igual que al alumnado } \\
\text { le ponen el hueco del plan de acción tutorial pues que por } \\
\text { ejemplo haya un espacio (...) donde tengamos la seguridad } \\
\text { de que tenemos una hora para trabajar, que no solamente se } \\
\text { beneficiaría este grupo, si no en general la facultad porque } \\
\text { hay más grupos en la facultad trabajando también proyectos } \\
\text { de innovación." }\end{array}$ \\
\hline
\end{tabular}

Tabla 8. Referencias referidas a propuestas de mejora. 
Por último, en cuanto a los resultados que se han percibido tras el desarrollo del proyecto se concretan en diferentes líneas. Por una parte su puesta en marcha ha fortalecido la coordinación entre profesorado que imparten las mismas asignaturas, pero que por pertenecer a distintos departamentos, hasta el momento no habían trabajado de manera colaborativa y coordinada, así como profesorado que imparte distintas asignaturas. Por otra parte, se ha percibido una mayor motivación del alumnado, mayor predisposición al trabajo y actitudes más colaborativas en las interactuaciones entre el alumnado.

Al mismo tiempo, la metodología y procedimientos seguidos para el desarrollo de las actividades, ha facilitado la oportunidad de debatir y reflexionar, más allá del temario establecido, vinculándolo además a lo que se hace en la Escuela, mediante el intercambio con centros, así como con alumnado de otras universidades que cursaban asignaturas afines.

\begin{tabular}{|c|c|c|c|}
\hline CATEGORÍA & $\begin{array}{c}\mathbf{N}^{0} \text { DE } \\
\text { REFERENCIAS }\end{array}$ & PARTICIPANTE & REFERENCIAS \\
\hline \multirow{4}{*}{$\begin{array}{l}\text { RESULTADOS } \\
\text { DEL PROYECTO }\end{array}$} & \multirow{4}{*}{16} & Docent7. G.D & $\begin{array}{l}\text { "El hecho de que los alumnos interactúen con otros, ya no solo } \\
\text { otro tipo de enseñanza, el hecho de sacarlos de alli y que vean otro } \\
\text { docente, otro profesor, otra persona que les comenta algo nuevo } \\
\text { sacándolo del espacio de confort del aula en el que se adecúan y } \\
\text { se habitúan y se acomodan mucho a la rutina. El sacarlos de ahí } \\
\text { y llevarlos a otra aula hace que despierten sus sentidos, entonces } \\
\text { colaboran más unos con otros, interactúan más y le da ese plus de } \\
\text { animosidad para interiorizar mejor los contenidos." }\end{array}$ \\
\hline & & Docent1. G.D & $\begin{array}{l}\text { "Algunos de los profesores que han hecho alli actividades habia } \\
\text { coincidido con gente que estaba trabajando o que estaban } \\
\text { comiendo y todo el mundo ha manifestado, que eso es una cosa } \\
\text { curiosa, que todo el mundo guardaba silencio. No habia bullicio } \\
\text { que impidiera desarrollar la actividad sino que todo el mundo } \\
\text { guardaba silencio y que incluso habia gente que estaba comiendo } \\
\text { o haciendo trabajos y en algún momento se mostraba interesada y } \\
\text { se levantaban a ver que estaban haciendo, con lo cual eso también } \\
\text { es significativo." }\end{array}$ \\
\hline & & Alum1. G.D & $\begin{array}{l}\text { "El tipo de carteles que ha estado colgado en el espacio es } \\
\text { diferente. (...) Ahora mismo puede encontrar otro tipo de } \\
\text { información también colgada en las paredes que yo creo que es } \\
\text { interesante y que no se hace y que muchas veces las producciones } \\
\text { que hace el alumnado se quedan entre el alumnado y el profesorado } \\
\text { y es un trabajo que se ha hecho, que ha costado un tiempo y no } \\
\text { se aprovecha. Así se está difundiendo información y conocimiento } \\
\text { utilizando el mobiliario y los espacios de la facultad. Yo creo que } \\
\text { también es algo interesante que ha traído el proyecto." }\end{array}$ \\
\hline & & Docent1. G.D & $\begin{array}{l}\text { Bueno pues ella su ponencia iba sobre eso, los beneficios que } \\
\text { plantea en los niños que son mellizos y tal y vino gente de fuera } \\
\text { de la facultad. Gente de fuera de la facultad se enteró que ella } \\
\text { trabajaba ese tema y vinieron porque les interesaba, familias, que } \\
\text { eso también es un plus de poder ofrecer actividades que sean de } \\
\text { interés." }\end{array}$ \\
\hline
\end{tabular}

Tabla 9. Referencias referidas a los resultados del proyecto. 
Igualmente, otro resultado apreciable del proyecto es la puesta en valor del espacio físico como un instrumento de aprendizaje a través del uso de las paredes de la facultad para exponer las producciones del alumnado, ayudando de esta forma a la difusión del conocimiento y otorgarle una mayor importancia al trabajo que elabora el alumnado en el contexto de las asignaturas. Además, en este sentido gracias a este proyecto se ha contribuido a la transferencia de conocimiento a la sociedad, sin duda un elemento clave y en muchas ocasiones una tarea pendiente de la Universidad.

\section{DISCUSIÓN Y CONCLUSIONES}

Tras la evaluación del proyecto emergen diferentes cuestiones que pueden ser de interés para la comunidad educativa de cara a la puesta en marcha de proyectos similares. Algunos de estos temas han versado sobre la formación, retos y desarrollo del profesorado. Sobre las implicaciones del espacio educativo en la motivación, participación y colaboración del alumnado, o la difusión y transferencia del conocimiento que se ha desprendido de la puesta en práctica del proyecto de innovación.

Para una mayor claridad en la exposición de las conclusiones, éstas se organizaran siguiendo los apartados expuestos en el apartado de resultados. Así, en cuanto a las valoraciones positivas, el trabajo desarrollado en el proyecto ha sido valorado como muy enriquecedor por parte del profesorado. Las valoraciones recibidas en este sentido reflejan que la experiencia ha hecho posible unir a docentes con intereses comunes que han encontrado nexos de unión, favoreciendo de esta forma la interdisciplinariedad, tan importante para los títulos que se imparten en la Facultad de Educación como defienden Spelt, Biemans, Tobi, Luning, y Mulder, (2009), Pozuelos, Rodríguez Miranda y Travé (2012), Botella y Hurtado (2017) o Lobato, Ladrón de Guevara, Adame, Soria y Salazar (2017). Esto se debe a que la metodología docente empleada, al tener lugar en un contexto espacial y en ocasiones, incluso temporal, fuera del aula tradicional, ha hecho posible la reflexión y participación activa del alumnado, al permitirles pensar y opinar sobre diversos temas relacionados con el ámbito educativo.

Vinculando las valoraciones negativas y las propuestas de mejoras que han ido unidas en la mayoría de los casos, se desprende también la necesidad de proporcionar tiempos al profesorado para que los equipos de trabajo puedan trabajar y evaluar sobre los proyectos de innovación como también defiende Paredes Labra (2004) o Bolarin-Martínez y Yus, (2015). Al mismo tiempo, como resultado de este proyecto, se ha reconocido la posibilidad de llevar a cabo la transferencia de conocimiento desde la universidad a la sociedad, una función que como destacan Álvarez y Osoro (2014) o López Martín (2017) se debe potenciar para incrementar el impacto social de las acciones y actividades que se llevan a cabo en la universidad.

\section{RECONOCIMIENTOS}

La realización de este trabajo forma parte del Proyecto COETUM: Proyecto para la creación de un espacio de ágora educativa, financiado en convocatoria pública competitiva por el Servicio de Orientación y Formación Docente de la Universidad de Extremadura. Este proyecto está dirigido 
por la Dra. Prudencia Gutiérrez Esteban y formado por los profesores y profesoras José Luis Torres Manano Rama Carvalho, María de las Mercedes Gómez Acuñas, Elia Fernández Díaz, Francisco de Paula Rodríguez Miranda, Luis Manuel Casas García, María Magdalena López Pérez, Lorea Fernández Olaskoaga, Juan Luis de la Montaña Conchiña, María Guadalupe de la Maya Retamar, José Luis Ramos Sánchez, Ana Isabel González Contreras, Estefanía Capilla Garrido, Vanessa Candil Recio, María Lourdes Hernández Rincón, Pilar Cañamero Sánchez, Zacarías Calzado Almodóvar, María Encarnación Masot Martínez, César Rina Simón, María José Rabazo Méndez, Rodrigo Espada Belmonte y Clara Báez Merino.

\section{REFERENCIAS}

Acar, H. (2014). Learning environments for children in outdoor spaces. Procedia-Social and Behavioral Sciences, 141, 846-853. DOI: https://doi.org/10.1016/j.sbspro.2014.05.147

Álvarez, C., y Osoro, J. M. (2014). Colaboración Universidad-Escuela para el cambio escolar. Una investigación-acción en proceso. Innovación educativa, 24, 215-227. DOI: https://doi. org/10.15304/ie.24.1483

Amann, B. (2016). Educación para el Desarrollo Sostenible (EDS) y arquitectura escolar. El espacio como reactivo del modelo pedagógico. Bordón. Revista de Pedagogía, 68(1), 145-164. DOI: https://doi.org/10.13042/Bordon.2016.68109

Boettcher, J. V. (2007). Ten core principles for designing effective learning environments: Insights from brain research and pedagogical theory. Innovate: Journal of Online Education, 3(3), $2-8$.

Bolarin-Martínez, M. J. y Yus, M. A. M. (2015). La coordinación docente en la universidad: retos y problemas a partir de Bolonia. Profesorado. Revista de Currículum y Formación de Profesorado, 19(2), 319-332.

Botella, A. M. y Hurtado, A. (2017). Innovación Educativa y renovación de metodologías docentes: Ieducarts y L'Hort 2.0. Innovación Educativa, 27, 205-217. Disponible en: http://www.usc. es/revistas/index.php/ie/article/view/4107 ISSN 2340-0056

Cobo, C. y Moravec, J. (2010). Invisible learning. Barcelona: Laboratori de Mitjans Interactius (University of Barcelona Press).

Cohen, B. (2010). Space to Develop: How Architecture Can Play a Vital Role in Young Children's Lives. París: CELE Exchange, Centre for Effective Learning Environments, 2010/06, OECD Publishing. DOI: http://dx.doi.org/10.1787/5kmbjxzzs9d6-en

Crespo, J. M. y Lorenzo, M. D. (2016). Los espacios de la escuela primaria inclusiva: conexiones y desarmonías entre la normativa de construcciones escolares y las finalidades del sistema educativo. Bordón. Revista de Pedagogía, 68(1), 131-144. DOI: https://doi.org/10.13042/ Bordon.2016.68108

Eberhard, J. P. (2009). Applying neuroscience to architecture. Neuron, 62(6), 753-756. DOI: https:// doi.org/10.1016/j.neuron.2009.06.001

Fernández-Díaz, E., Gutiérrez-Esteban, P. y Fernández-Olaskoaga, L. (2015). Una investigación colaborativa interuniversitaria para repensar un modelo docente comprometido con el cambio educativo. Revista interuniversitaria de formación del profesorado, 82, 143-156. 
Guldbaek, J., H. B. Vinkel y M. G. Broens (2011). Transforming Pedagogical Ethos into an Effective Learning Environment. París: CELE Exchange, Centre for Effective Learning Environments, 2011/03, OECD Publishing. DOI: http://dx.doi.org/10.1787/5kgdzvmgvjzn-en

Guney, A. y Al, S. (2012). Effective learning environments in relation to different learning theories. Procedia-Social and Behavioral Sciences, 46, 2334-2338. DOI: https://doi.org/10.1016/j. sbspro.2012.05.480

Heppell, S., Chapman, C., Millwood, R., Constable, M. y Furness, J. (2004). Building learning futures. A research project at Ultralab within the CABE/RIBA. Building Futures programme. Chelmsford, UK: Ultralab.

Hernando Calvo, A. (2015). Viaje a la escuela del siglo XXI. Madrid: Fundación Telefónica.

Ibrahim, M. S., Razak, A. Z. A. y Kenayathulla, H. B. (2013). Smart principals and smart schools. Procedia-Social and Behavioral Sciences, 103, 826-836. DOI: https://doi.org/10.1016/j.sbspro.2013.10.404

Kuuskorpi, M. y Cabellos González, N. (2011). The Future of the Physical Learning Environment: School Facilities that Support the User. París: CELE Exchange, Centre for Effective Learning Environments, 2011/11, OECD Publishing. DOI: http://dx.doi.org/10.1787/5kg0lkz2d9f2-en

Lobato, M. J., Ladrón de Guevara, L., Adame, Z., Soria, A. y Salazar, M. D. C. (2017). Una propuesta de proyecto interdisciplinar en la formación inicial del profesorado de Educación Infantil. Simposio Internacional El Desafio de Emprender en la Escuela del Siglo XXI (2017), pp. 70-84.

López Martín, R. (2017). Hacia una innovación docente de calidad de la educación superior. Claves para la reflexión. Foro educacional, 28, 11-28.

Mokthar, F., Jiménez Rodríguez, M. Á., Heppell, S. y Segovia, N. (2016). Creando espacios de aprendizaje con los alumnos para el tercer milenio. Bordón. Revista de pedagogía, 68(1), 61-82. DOI: https://doi.org/10.13042/Bordon.2016.68104

Muñoz-Rodríguez, J. M. y Olmos, S. (2016). Naturaleza social de la arquitectura y educación. Un estudio descriptivo. Bordón. Revista de Pedagogía, 68(1), 99-114. DOI: https://doi. org/10.13042/Bordon.2016.68106

Olivos, P. (2010). Ambientes escolares. En J. I. Aragonés y M. Amérigo (Eds.), Psicología Ambiental (pp. 205-222). Madrid: Pirámide. 3 ed.

Paredes Labra, J. (2004). Cultura escolar y resistencia al cambio. Tendencias pedagógicas, 9, 131142.

Pozuelos, F. J., Rodríguez Miranda, F. D. P., y Travé, G. (2012). El enfoque interdisciplinar en la enseñanza universitaria y aprendizaje basado en la investigación. Un estudio de caso en el marco de la formación. Revista de educación, 357, 213-214.

Remess, M. y Winfield, F. N. (2008). Espacios educativos y desarrollo: alternativas desde la sustentabilidad y la regionalización. Investigación y Ciencia, 16(42), 45-50.

Rigolon, A. (2010). European Design Types for $21^{\text {st }}$ Century Schools: An Overview. París: CELE Exchange, Centre for Effective Learning Environments, 2010/03, OECD Publishing. DOI: http://dx.doi.org/10.1787/5kmh36gpvmbx-en 
Rute, A. (2010). Diálogos e perspetivas de jovens sobre o ambiente construído da escola. Porto, Faculdade de Psicologia e Ciências da Educação, Universidade do Porto. Tese de Doutoramento.

Spelt, E. J., Biemans, H. J., Tobi, H., Luning, P. A. y Mulder, M. (2009). Teaching and learning in interdisciplinary higher education: A systematic review. Educational Psychology Review, 21(4), 365-378. DOI: https://doi.org/10.1007/s10648-009-9113-z

Thornburg, D. D. (2004). Campfires in cyberspace. Instructional technology, 1(10), 3-10.

Ultralab (2004). Building Learning Futures. London: CABE/RIBA. 